\title{
Japan's optical/infrared astronomy plan
}

\author{
Masanori Iye \\ National Astronomical Observatory, Mitaka, Tokyo 181-8588, Japan \\ email: iye@optik.mtk.nao.ac.jp
}

\begin{abstract}
The status of Japan's planning of the optical/infrared astronomy projects for the 2010 's is briefly reviewed. The road map shows a $30 \mathrm{~m}$ class extremely large ground based telescope project with advanced adaptive optics capability, JELT, and a mid-infrared optimized $3.5 \mathrm{~m}$ space telescope project, SPICA, as the top-priority major project to be accomplished probably on international collaboration basis.
\end{abstract}

Keywords. Akari, ELT, SPICA, Subaru

\section{Status of the projects}

The optical/infrared astronomical community of Japan conducted a two-year study and produced a report booklet in 2005 to recommend major optical/infrared astronomy missions to be accomplished in the 2010 decade. The report expects to complete JELT, an extremely large telescope of about $30 \mathrm{~m}$ in diameter with innovative adaptive optics capability, to make advances in observational capabilities to investigate various newly emerging science problems as well as ever standing key questions. It also identifies SPICA, a $3.5 \mathrm{~m}$ astronomical space telescope project optimized for far-infrared regime, as the key space mission for Japan's optical/infrared astronomy community to accomplish. SPICA will bridge the wavelength gap between those covered by ALMA and JWST/Herschel.

JELT and SPICA are natural missions advancing the expertise cultivated around $8.2 \mathrm{~m}$ Subaru Telescope and $60 \mathrm{~cm}$ Akari satellite, respectively. There are communities heritage on engineering and science around these leading projects.

As for the design and development efforts on JELT, the core group is making some generic researches on: $(i)$ new zero-expansion ceramic material to be used for next generation mirrors; ( $i i)$ high-precision grinding method to figure out aspheric surface to shape error less than a wavelength; and (iii) developments for new methodology sensors. There is a concrete plan to construct a $3.5 \mathrm{~m}$ telescope with circularly segmented mirrors to prove these new technologies. National Astronomical Observatory is especially interested in promoting the JELT on Mauna Kea to make good use of Subaru heritage in people and facilities.

SPICA would achieve the highest sensitivity among proposed missions in the wavelength range $15-130 \mu \mathrm{m}$ and could be a mission complementary to Herschel and JWST. SPICA will be placed at an L2 orbit to avoid thermal background from the Sun and the Earth. Research and development for $(i)$ light-weight mirrors, (ii) cryogenic system with radiative cooling, and (iii) science instruments, are under investigation.

Additional space missions under conceptual phase are JASMINE, a space telescope for astrometry to map out Galactic bulge structure and JTPF, a mission to study extra-solar planets.

National funding policy on basic science is not in highly encouraging situation and costly projects are facing financial difficulties despite well received publicity of astronomical achievements. 


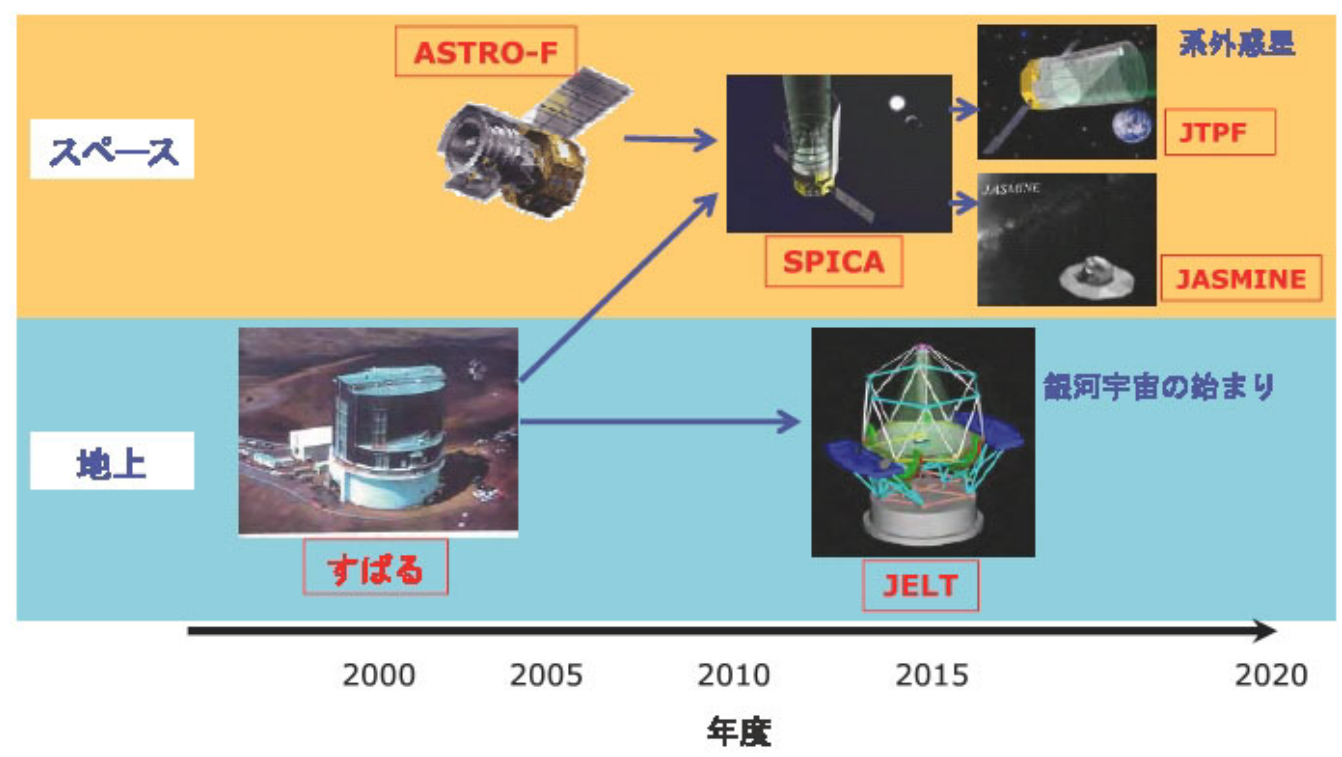

Figure 1. Road map of Japan's optical/infrared astronomy of 2010s.

Since the required budgetary scale of these ambitious missions is likely to be beyond the level any single nation can fully support, organizing an international consortium to accomplish these science missions will be extremely important. In this regard, positive discussion toward international coordination for common goals is essential and the present Special Session in the IAU XXVI General Assembly, for instance, is highly useful for those involved groups.

The relevant URLs for further information are:

JELT home page: <http://jelt.mtk.nao.ac.jp/index_e.html>, and

SPICA mission: <http://www.ir.isas.jaxa.jp/SPICA/index.html>.

\section{References}

Iye, M. 2006, in: P. A. Whitelock, M. Dennefeld \& B. Leibundgut (eds.), The Scientific Requirements for Extremely Large Telescopes, Proc. IAU Symp. No. 232 (Cambridge: CUP), p. 381

Onaka, T., \& Nakagawa, T. 2005, Adv. Space Res. 36, 1123 